\title{
Classical Many-Body Time Crystals
}

\section{Journal Article}

\section{Author(s):}

Heugel, Toni L.; Oscity, Matthias; Eichler, Alexander; Zilberberg, Oded; Chitra, Ramasubramanian

Publication date:

2019-09-19

Permanent link:

https://doi.org/10.3929/ethz-b-000367966

\section{Rights / license:}

In Copyright - Non-Commercial Use Permitted

\section{Originally published in:}

Physical Review Letters 123(12), https://doi.org/10.1103/PhysRevLett.123.124301

\section{Funding acknowledgement:}

177198 - Zeptonewton force sensing on a membrane resonator platform (SNF)

163818 - Electronic and photonic quantum engineered systems (SNF) 


\title{
Classical Many-Body Time Crystals
}

\author{
Toni L. Heugel, ${ }^{1, *}$ Matthias Oscity, ${ }^{1,{ }^{*} \dagger}$ Alexander Eichler, ${ }^{2}$ Oded Zilberberg $\odot,{ }^{1}$ and R. Chitra ${ }^{1}$ \\ ${ }^{1}$ Institute for Theoretical Physics, ETH Zurich, 8093 Zurich, Switzerland \\ ${ }^{2}$ Institute for Solid State Physics, ETH Zurich, 8093 Zurich, Switzerland
}

(Received 6 March 2019; published 19 September 2019)

\begin{abstract}
Discrete time crystals are a many-body state of matter where the extensive system's dynamics are slower than the forces acting on it. Nowadays, there is a growing debate regarding the specific properties required to demonstrate such a many-body state, alongside several experimental realizations. In this work, we provide a simple and pedagogical framework by which to obtain many-body time crystals using parametrically coupled resonators. In our analysis, we use classical period-doubling bifurcation theory and present a clear distinction between single-mode time-translation symmetry breaking and a situation where an extensive number of degrees of freedom undergo the transition. We experimentally demonstrate this paradigm using coupled mechanical oscillators, thus providing a clear route for time crystal realizations in real materials.
\end{abstract}

DOI: 10.1103/PhysRevLett.123.124301

In periodically modulated nonlinear systems, discrete time-translation symmetry can be spontaneously broken, leading to inherently slower dynamics than that of the drive [1-6]. A rapidly expanding community is principally focused on such a phenomenon in periodically driven closed quantum systems, where disorder and interactions are considered to be essential for so-called discrete time crystals [7-20]. A time-crystalline phase of matter stabilized by many-body localization was first observed in a onedimensional trapped-ion system [13]. Surprisingly, time crystals were also seen in three-dimensional ensembles of NV centers [14] and in spin- $1 / 2$ nuclei in phosphate materials [21] where disorder-induced localization effects are absent. The latter results indicate a wider class of time-crystalline behavior, including classical counterparts [22-27].

A natural arena for realizing time crystals is provided by parametric resonators. A parametrically pumped resonator mode plays an important role in many areas of science and technology. In its best-known form, parametric pumping describes the modulation of a resonator's potential at twice its natural frequency [1-4]. When the modulation depth exceeds an instability threshold, the resonator undergoes a period-doubling bifurcation to a new regime stabilized by nonlinearities [1]. This timetranslation symmetry breaking (TTSB) leads to two stable parametric phase states that have equal amplitude, opposite phase, and half the oscillation frequency of the parametric drive [17,28-30]. Interestingly, these states can be associated with two states of a classical bit [31-35] or with an Ising spin [28-30,36-46]. Networks of such coupled resonators have been proposed as simulation platforms for complex Ising-like models that are very hard to solve with conventional computers [17,36-43]. (a)

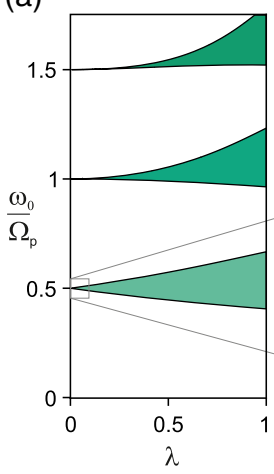

(b)

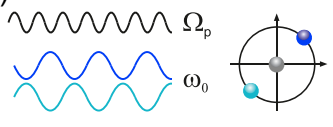

(c)

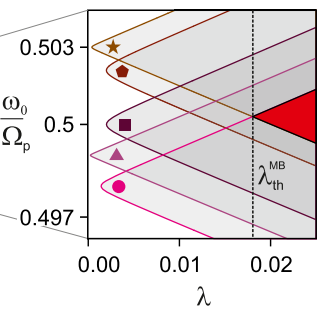

(d)

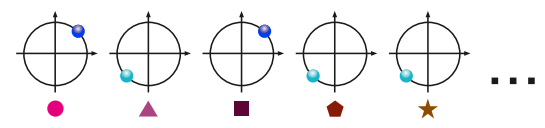

FIG. 1. (a) Stability diagram of a single parametrically driven resonator mode with eigenfrequency $\omega_{0}$ as a function of parametric pumping frequency and depth, $\Omega_{p}$ and $\lambda$, respectively [1,48]; cf. Eq. (1). Green tongue shapes indicate regions where the linear resonator becomes unstable. (b) Beyond the instability threshold in the first lobe, $\Omega_{p} \sim 2 \omega_{0}$, the nonlinear resonator undergoes a period-doubling bifurcation and oscillates with frequency $\Omega_{p} / 2$. Because of the period doubling, there exist two possible phase states with equal amplitude but opposite phase. In some frequency ranges, there is an additional zerodisplacement stable solution. (c) Enlargement of the stability diagram around the first instability lobe for $N$ coupled resonators. Here, $N$ nondegenerate normal modes (marked by different color and symbols) generically arise; cf. Eq. (1). The red area indicates the region of many-body TTSB. (d) Inside the many-body TTSB, each of the normal modes reside in one of the phase states. The resulting multistate configuration depends on the coupling coefficients $\beta_{i j}$, the nonlinearities, and noise fluctuations. 
In this work, we show that a many-body TTSB can be easily realized in a classical network of dissipative parametric resonators. We present a general theoretical analysis and derive conditions for the manifestation of many-body TTSB in this system. This is complemented by a simple tabletop experimental demonstration using two coupled resonators. Our setup allows us to tune the coupling strength, and we find a regime where the modes of the system jointly undergo TTSB into well-defined parametric phase state configurations. Our experiment thus realizes the simplest building block that highlights the plethora of accessible TTSB solutions. At the same time, we test our understanding of the general many-body model against a well-controlled and accessible experimental implementation. Our work lifts the ambiguity surrounding the concept of time crystals by establishing sufficient conditions for their generation.

We consider a classical network of $N$ coupled nonlinear parametric oscillators, whose dynamics is governed by $N$ equations of motion

$$
\begin{aligned}
\ddot{x}_{i} & +\omega_{i}^{2}\left[1-\lambda \cos \left(\Omega_{p} t\right)\right] x_{i}+\gamma_{i} \dot{x}_{i} \\
& +\alpha_{i} x_{i}^{3}+\eta_{i} x_{i}^{2} \dot{x}_{i}-\sum_{i \neq j} \beta_{i j}^{2} x_{j}=0,
\end{aligned}
$$

where dots mark differentiations with respect to time $t, x_{i}$ is the displacement, $\omega_{i}$ the eigenfrequency, $\gamma_{i}$ the dissipation, $\alpha_{i}$ the quartic nonlinearity, and $\eta_{i}$ the nonlinear damping of the $i$ th mode. The system is excited by a single parametric pump of modulation depth $\lambda$ and frequency $\Omega_{p}$. Each mode $i$ couples to other modes $j \neq i$ in the form of a driving force in proportion to $x_{i}$ and with a coupling coefficient $\beta_{i j}^{2}$.

We can perturbatively solve the system using the slowflow method [47]: we rewrite Eq. (1) as $2 N$ first-order differential equations and perform a van der Pol transformation with frequency $\omega=\Omega_{p} / 2$, followed by time averaging, to obtain the slow-flow equation

$$
\dot{\boldsymbol{X}}=A(\boldsymbol{X}) \boldsymbol{X},
$$

where $\boldsymbol{X}=\left(u_{1}, v_{1}, u_{2}, v_{2}, \ldots, u_{N}, v_{N}\right)^{T}$, with $u_{i}$ and $v_{i}$ the slowly varying phase-space quadratures of the individual resonators. This equation is valid if the dimensionless quantities $1-\left(\omega / \omega_{i}\right)^{2}, \lambda, \gamma_{i} / \omega_{i},\left(\eta_{i} / \omega_{i}\right) x_{i}^{2}, \beta_{i j}^{2} / \omega_{i}^{2}$, and $\left(\alpha_{i} / \omega_{i}^{2}\right) x_{i}^{2}$ are of order $\epsilon$, where $0<\epsilon \ll 1$ [47]. These conditions are easily satisfied for a network of nearly identical oscillators. The matrix $A$ can be written as

$$
A=\left(\begin{array}{cccc}
a_{1}(\boldsymbol{X}) & b_{12} & \ldots & b_{1 N} \\
b_{1,2} & a_{2}(\boldsymbol{X}) & \ddots & \vdots \\
\vdots & \ddots & \ddots & b_{(N-1) N} \\
b_{1 N} & \cdots & b_{(N-1) N} & a_{N}(\boldsymbol{X})
\end{array}\right),
$$

where the $a_{i}$ and $b_{i j}$ are given by

$$
a_{i}(\boldsymbol{X})=-\frac{1}{8 \omega}\left(\begin{array}{cc}
a_{i, 1} & a_{i,+} \\
a_{i,-} & a_{i, 1}
\end{array}\right), \quad b_{i j}=\left(\begin{array}{cc}
0 & \frac{\beta_{i j}^{2}}{2 \omega} \\
-\frac{\beta_{i j}^{2}}{2 \omega} & 0
\end{array}\right),
$$

with $i \neq j$ and $i, j=1,2, \ldots, N$, and using the definitions $a_{i, 1}=4 \gamma_{i} \omega+\eta_{i} \omega X_{i}^{2}, a_{i, \pm}=2\left(\lambda \omega_{i}^{2}+2\left(\omega_{i}^{2}-\omega^{2}\right)\right) \pm 3 \alpha_{i} X_{i}^{2}$, and $X_{i}^{2}=u_{i}^{2}+v_{i}^{2}$. In general, the number of steady-state solutions, both stable and unstable, to this $\mathrm{N}$-body problem varies from 1 to $5^{N}$ depending on the parameter regimes [28].

In the absence of nonlinearities, $\alpha_{i}=\eta_{i}=0$, the natural description of the resonator network is given by $N$ normal modes with eigenfrequencies $\nu_{k}, k=1, \ldots, N$. The dynamics of the normal modes is determined by the eigenvalues and the eigenvectors of $A$. The $2 N$ eigenvectors define the positions and momenta of the $N$ normal modes. The time evolution of the $k$ th normal mode is given by $e^{\Lambda_{k} t}$, with $\Lambda_{k}$ the respective eigenvalue. The motion will be bounded for negative $\operatorname{Re}\left\{\Lambda_{k}\right\}$ and manifest parametric instability, i.e., unbounded dynamics when $\operatorname{Re}\left\{\Lambda_{k}\right\}>0$. Each normal mode exhibits a corresponding parametric stability phase diagram known as "Arnold tongues," delineating regions where dissipation stabilizes the motion and regions where the linear system shows unbounded dynamics, see Fig. 1(a). In the following, we will focus on the dominant instability lobe occurring around $\Omega_{p} \sim 2 \nu_{k}$, when the parametric drive exceeds a threshold $\lambda \geq \lambda_{\mathrm{th}}^{k}$ [48].

In general, it is not dissipation but the underlying nonlinearities $\left(\alpha_{i}, \eta_{i}\right)$ that stabilize the normal-mode oscillations against unbounded growth [5]. The classical systems we consider here are therefore no more limited by heating than their quantum counterparts. At the boundary of its main instability lobe, each normal mode undergoes a period-doubling bifurcation alongside a spontaneous $Z_{2}$ symmetry breaking between the two parametric phase states; see Fig. 1(b). This is a simple manifestation of TTSB in the steady state of an effective single parametric mode. It is important to note that although a single normal mode can involve an extensive number of resonators of the network, it does not give rise to a many-body TTSB because it does not involve an extensive number of independent degrees of freedom.

A many-body TTSB phase is realized in the resonator network in a region where an extensive number of normal modes undergo the aforementioned period-doubling transition. A simple recipe to realize a many-body TTSB consists of finding the parametric pumping amplitude $\lambda_{\mathrm{th}}^{\mathrm{MB}}\left(\omega_{P}\right)=\min _{\lambda}\left\{\lambda>\lambda_{\mathrm{th}}^{k}\left(\omega_{P}\right), \forall k\right\}$ at which all normal modes are driven above their respective instability thresholds, see Fig. 1(c). There, each normal mode finds itself in a parametric phase state, see Fig. 1(d). Note that the manybody threshold holds in the limit of weak nonlinearities and does not include corrections stemming from nonlinear internormal mode coupling. We have numerically verified 


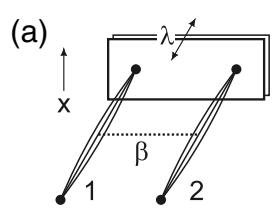

(b)

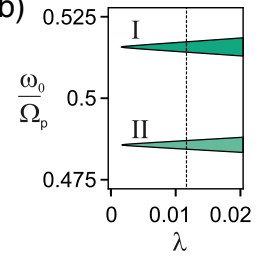

(c)

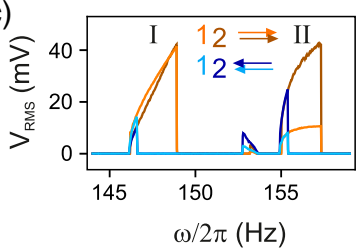

(d)

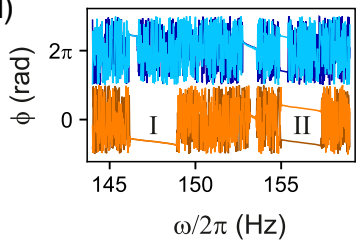

(e)

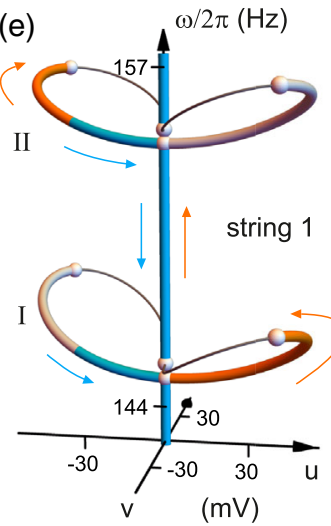

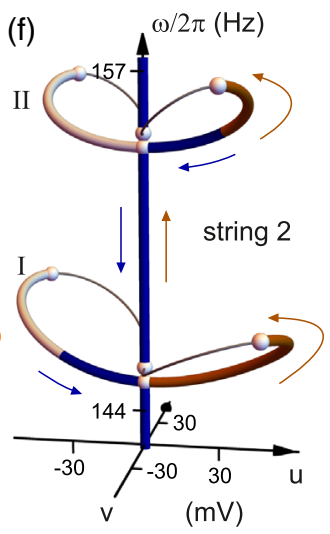

FIG. 2. Strongly coupled oscillators: (a) Schematic setup representing two parametrically driven strings coupled via an additional mechanical connection. (b) Calculated normal-mode stability diagram of the symmetric (I) and antisymmetric (II) eigenmodes of the coupled system. (c) Measured amplitude and (d) phase of strings 1 and 2 for the up sweep (orange and brown) and down sweep (light and dark blue) where both oscillators are parametrically driven at frequency $2 \omega$. The fixed phase relations in regions I and II are signatures of the corresponding normal mode symmetries. (e)-(f) Simulated steady-state solutions of oscillators 1 and 2 in the rotating frame phase space $(u, v)$ calculated from the slow-flow equations, cf. Eq. (2) as a function of $\omega$. The thick (thin) tubes are stable (unstable) solutions and white spheres indicate the positions of bifurcations. The stable branches corresponding to the experiment are highlighted in matching colors for up- and down sweeps. Arrows indicate the sweep directions. In agreement with the experiment, fixed symmetric and antisymmetric phase relations appear in the normal modes I and II, respectively.

that this picture persists beyond the regime of weak nonlinearities. For stronger nonlinearities, the system is predicted to undergo a cascade of period-doubling bifurcations resulting in chaotic dynamics [49-51]. In the meanfield limit of $N$ identical resonators, i.e., $\omega_{i} \equiv \omega_{0}$ and $\gamma_{i}=\gamma$, with all-to-all coupling $\beta_{i j}=\beta / \sqrt{N}$, apart from the symmetric mode, all other instability lobes coincide with that of the antisymmetric $(a)$ mode. The respective instability thresholds $\left(\lambda>\lambda_{\text {th }}\right)$ are given by [52]

$\lambda_{\mathrm{th}}^{s / a}=\frac{4 \omega}{\omega_{0}^{2}} \sqrt{\frac{\gamma^{2}}{4}+\left(\frac{\omega^{2}-\omega_{0}^{2}}{2 \omega}+\left\{\begin{array}{ll}\frac{(N-1)}{N}, & s \\ \frac{-1}{N}, & a\end{array}\right\} \frac{\beta^{2}}{2 \omega}\right)^{2}}$.

The overlap region of $\lambda_{\mathrm{th}}^{s / a}$ defines $\lambda \geq \lambda_{\mathrm{th}}^{\mathrm{MB}}\left(\Omega_{p}\right)$.

In the following we discuss two limits, "strong" and "weak" coupling, that are defined relative to the parametric modulation strength $\lambda$. Since all the oscillators are driven with the same periodic drive, they will synchronize into one of $2^{N}$ to $3^{N}$ phase state configurations: for weak $\beta_{i j}^{2}$, these correspond to the possible compositions of the $N$ individual resonators [17,53], while for strong $\beta_{i j}^{2}$, they correspond to the configurations of collective normal modes. In both cases, all these states manifest TTSB and the chosen configuration will depend on initial conditions, noise, and the strength of the nonlinearities. Noise can induce transitions between phase states. However, for detunings within the multimode instability lobe, transitions occur only between TTSB states and are exponentially suppressed $[54,55]$. To summarize, we predict that an array of coupled dissipative parametric resonators realizes a stable TTSB phase in its steady state. This phase endures in a wide region of parameter space and is robust to fluctuations.

We now report on an experimental demonstration of many-body TTSB in a system of two coupled mechanical modes. Our setup is based on the lowest transverse vibrational modes of two macroscopic strings. The strings are clamped onto a fixed frame at one end, while the other end is attached to a stiff plate that has two purposes; first, the plate can be driven into vibrations parallel to the string axes by an electric motor. These vibrations modulate the tension inside the strings and generate parametric pumping of both string modes. Second, the plate transmits vibrations between the strings, which leads to weak intrinsic coupling between the modes. In some experiments, we introduce strong mode coupling by way of a mechanical connection close to the mode antinodes, see Fig. 2(a).

The motion of each string is independently measured with a dedicated piezo detector embedded into one clamping point. We use a lock-in amplifier (Zurich Instruments HF2LI) to actuate plate vibrations and to read out the electrical signals from the two piezo detectors, which are proportional to the strings' displacements. All measurements in this work were carried out in the form of frequency sweeps, where the actuation frequency $\Omega_{p}=2 \omega$ and the detection frequency $\omega$ were swept slowly to capture the steady-state response of the modes. The measured string vibration amplitude at each frequency is proportional to the root-mean-square voltage $V_{\text {rms }}$ detected by the lock-in amplifier [52].

We use weak external driving for calibration of the modes, as outlined in Refs. [29,30]. In these experiments, the vibration amplitude is kept low and the influence of the intrinsic coupling is negligible. From the Lorentzian 


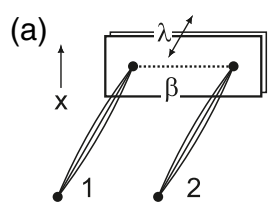

(b)

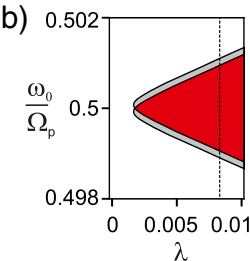

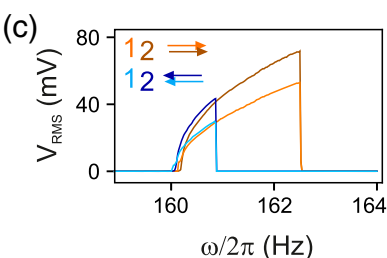

(d)

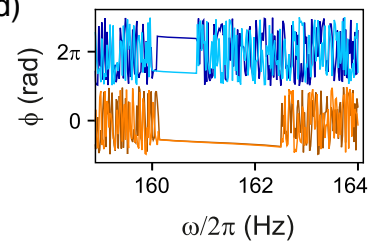

(e)

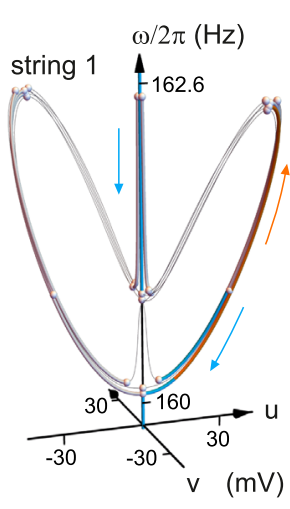

(f)

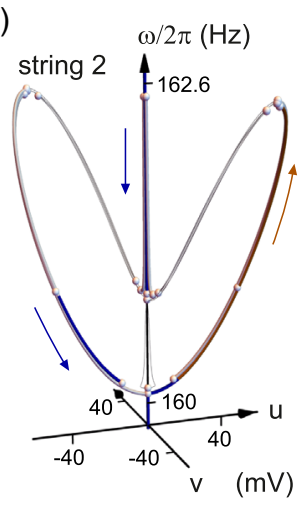

FIG. 3. Weakly coupled oscillators: (a) Schematic setup representing two parametrically driven strings weakly coupled via the driving plate. (b) Normal mode stability diagram. (c) Amplitude and (d) phase of strings 1 and 2 for the up sweep (orange and brown) and down sweep (light and dark blue) where both oscillators are parametrically driven at frequency $2 \omega$. Both symmetric and antisymmetric stable phase relations are observed depending on the system path in parameter space. This is the signature of a many-body time crystal. (e) and (f) The simulated steady-state solutions of oscillators 1 and 2 in the rotating-frame phase space $(u, v)$ calculated from the slow-flow equations, cf. Eq. (2) as a function of $\omega$. The thin tubes are unstable solutions and all other colored tubes represent stable solutions. The white spheres in these plots denote bifurcations. In agreement with the experiment, either symmetric or antisymmetric phase relations appear depending on the sweep direction (indicated by arrows).

response of each mode, we extract typical values for $\omega_{1,2} / 2 \pi=155 \pm 10 \mathrm{~Hz}$ (depending on ambient temperature) and $Q_{1,2} \sim 1200$. The effective mass $m=4.14 \times$ $10^{-4} \mathrm{~kg}$ is calculated from the geometry of the strings. By fitting to the large-amplitude response under strong parametric pumping, we obtain the coefficients of the nonlinear potential term, $\alpha_{1}=11.93$ and $\alpha_{2}=6.24 \mathrm{mV}_{\mathrm{rms}}^{-2} \mathrm{~s}^{-2}$, as well as those of the nonlinear damping, $\eta_{1}=7.1$ and $\eta_{2}=$ $3.9 \mu \mathrm{V}_{\mathrm{rms}}^{-2} \mathrm{~s}^{-1}$ (in the strong coupling case, we find $\eta_{1}=$ 3.55 and $\eta_{2}=1.95 \mu \mathrm{V}_{\mathrm{rms}}^{-2} \mathrm{~s}^{-1}$ ) [52]. Finally, in the presence of strong coupling, we use the normal mode frequency splitting to estimate $\beta=36.2 \pm 0.1 \mathrm{~Hz}$.

Strong coupling.-[Fig. 2(a)]: we first explore the regime where the two instability lobes corresponding to the symmetric and antisymmetric normal modes are well separated, see Fig. 2(b). In Figs. 2(c) and 2(d), we show the measured amplitudes and phases of both strings under a common parametric modulation as a function of frequency $\omega / 2 \pi$, respectively. As the frequency is slowly swept upwards, both resonators oscillate with the same phase from 146 up to $149 \mathrm{~Hz}$, while they are in opposing phase states from 155 up to $157.5 \mathrm{~Hz}$. The modes exhibit identical symmetries (s/a) when the frequency is swept downwards. These qualitative observations were consistent over many sweeps. The small peaks around $\omega / 2 \pi=153 \mathrm{~Hz}$ correspond to an unidentified eigenmode in the experimental setup that does not appear to affect the modes of interest.

We model the system with Eq. (2) for $N=2$ using the parameters extracted from the experiment. The results of our calculations provide a simple understanding of the experimental observations: as the frequency is swept, either the symmetric or antisymmetric normal modes undergo TTSB at their respective instability thresholds, recreating the effective single-mode TTSB discussed earlier. The coupling between the normal modes induced by nonlinearities is irrelevant in this regime as one mode is strongly off-resonant with the other. The experimental results are well described by the phase-space bifurcation diagrams for each resonator plotted in Figs. 2(e) and 2(f). Despite the fact that both resonators participate in the TTSB of the symmetric or antisymmetric modes, many-body TTSB is not observed in this strong-coupling limit as the two instability lobes do not overlap for experimentally accessible parametric excitation strengths.

Weak coupling.-Next, we remove the connection between the strings and rely on the driving plate to provide weak coupling between the string modes [Fig. 3(a)-3(b)]. The experimental data look very different in this regime [Fig. 3(c)-3(d)]. Both strings have nearly identical natural frequencies (within $50 \mathrm{mHz}$ from each other) and exhibit hysteresis when sweeping the frequency upwards and downwards. The frequencies where the oscillation drops to zero (during up sweeps) or jumps to a finite amplitude (during down sweeps) are precisely the same for both resonators. The strings oscillate in phase during the upsweep and out of phase during the down sweep. All of these features were reproduced over many sweeps.

The theoretical model corresponds to normal modes that are split by a very small coupling $\beta$, such that their instability lobes overlap strongly [Fig. 3(b)]. Since both normal modes exhibit TTSB and are weakly coupled by nonlinearities, we witness the realization of two-body TTSB. As before, the experimental results for the amplitude and phase are consistently explained by the weak coupling bifurcation diagram for both strings shown in Figs. 3(e)-3(f). In comparison with the strong coupling scenario of Figs. 2(e)-2(f), the weakly coupled system exhibits richer 
behavior. The selection of symmetric and antisymmetric solutions as a function of the sweeping direction may be explained in terms of the phase response of a linear resonator to a periodic external force. Below its natural frequency, a harmonic resonator oscillates with almost no phase lag in response to an external force. As the two string modes drive each other, they prefer to move in phase. In contrast, since the harmonic resonator response has a phase lag of $\sim \pi$ above the natural frequency, the string modes preferably oscillate out of phase during the down sweep. This many-body TTSB state is stable against small detunings $\omega_{1} \neq \omega_{2}$ and robust to noise (as seen in the experiment). Increasing noise levels are expected to preserve the underlying TTSB, but to induce transitions between the different stable solutions. For the inherent noise in our setup, we estimate a negligible transition rate of $\sim 10^{-5}-10^{-10} \mathrm{~Hz}$ [54].

Coupled parametric resonators provide the simplest, albeit rich platform to realize many-body discrete TTSB. Our paradigm of a many-body time crystal is straightforwardly generalizable to quantum resonator networks [38,56-59]. Higher-period TTSB is also realizable in these systems through a judicious choice of modulated nonlinearities [60-62]. The simple view of TTSB proposed in this Letter can be putatively applied to the recently seen TTSB in quantum spin systems [13,14]. Heuristically, the many-body spin system can be viewed as composed of multiple effective modes describing the eigenstates of the system. Depending on how many such modes undergo TTSB, single or many-mode TTSB will manifest in such spin system. An important distinction is that while noise and other imperfections lead to a decay of the TTSB state to a time translation symmetry unbroken state in the spin system $[13,14]$, they preserve TTSB in our resonator platform. Further investigations along this line are pending. To conclude, we provide a simple paradigm of many-body TTSB, which is applicable to a plethora of systems.

This work received financial support from the Swiss National Science Foundation through Grants (CRSII5_177198/1) and (PP00P2_163818).

"These authors contributed equally.

"Present address: Fachhochschule Nordwestschweiz FHNW, 5210 Windisch, Switzerland.

[1] L. Landau and E. Lifshitz, Mechanics, 3rd ed. (ButterworthHeinemann, Oxford, 1982), https://doi.org/10.1016/C20090-25569-3.

[2] M. Faraday, Phil. Trans. R. Soc. London 121, 299 (1831).

[3] E. Mathieu, J. Math. Pure Appl. 13, 137 (1868).

[4] Rayleigh, Philos. Mag. 24, 145 (1887).

[5] A. H. Nayfeh and D. T. Mook, Nonlinear Oscillations (Wiley-VCH Verlag GmbH, Weinheim, 2007).

[6] M. Dykman, Fluctuating Nonlinear Oscillators: From Nanomechanics to Quantum Superconducting Circuits (Oxford University Press, Oxford, 2012).
[7] V. Khemani, A. Lazarides, R. Moessner, and S. L. Sondhi, Phys. Rev. Lett. 116, 250401 (2016).

[8] D. V. Else, B. Bauer, and C. Nayak, Phys. Rev. Lett. 117, 090402 (2016).

[9] D. V. Else, B. Bauer, and C. Nayak, Phys. Rev. X 7, 011026 (2017).

[10] C. W. von Keyserlingk and S. L. Sondhi, Phys. Rev. B 93, 245146 (2016).

[11] C. W. von Keyserlingk, V. Khemani, and S. L. Sondhi, Phys. Rev. B 94, 085112 (2016).

[12] N. Y. Yao, A. C. Potter, I.-D. Potirniche, and A. Vishwanath, Phys. Rev. Lett. 118, 030401 (2017).

[13] J. Zhang, P. W. Hess, A. Kyprianidis, P. Becker, A. Lee, J. Smith, G. Pagano, I.-D. Potirniche, A. C. Potter, A. Vishwanath, N. Y. Yao, and C. Monroe, Nature (London) 543, 217 (2017).

[14] S. Choi, J. Choi, R. Landig, G. Kucsko, H. Zhou, J. Isoya, F. Jelezko, S. Onoda, H. Sumiya, V. Khemani, C. von Keyserlingk, N. Y. Yao, E. Demler, and M. D. Lukin, Nature (London) 543, 221 (2017).

[15] W. W. Ho, S. Choi, M. D. Lukin, and D. A. Abanin, Phys. Rev. Lett. 119, 010602 (2017).

[16] K. Sacha and J. Zakrzewski, Rep. Prog. Phys. 81, 016401 (2018).

[17] M. I. Dykman, C. Bruder, N. Lörch, and Y. Zhang, Phys. Rev. B 98, 195444 (2018).

[18] J. Rovny, R. L. Blum, and S. E. Barrett, Phys. Rev. B 97, 184301 (2018).

[19] W. Berdanier, M. Kolodrubetz, S. A. Parameswaran, and R. Vasseur, Proc. Natl. Acad. Sci. U.S.A. 115, 9491 (2018).

[20] N. Y. Yao and C. Nayak, Phys. Today 71, No. 9, 40 (2018).

[21] J. Rovny, R. L. Blum, and S. E. Barrett, Phys. Rev. Lett. 120, 180603 (2018).

[22] R. E. Goldstein, Phys. Today 71, No. 9, 32 (2018).

[23] N. Y. Yao, C. Nayak, L. Balents, and M. P. Zaletel, arXiv: 1801.02628 .

[24] F. M. Gambetta, F. Carollo, M. Marcuzzi, J. P. Garrahan, and I. Lesanovsky, Phys. Rev. Lett. 122, 015701 (2019).

[25] J. Smits, L. Liao, H. T. C. Stoof, and P. van der Straten, Phys. Rev. Lett. 121, 185301 (2018).

[26] L. Liao, J. Smits, P. van der Straten, and H. T. C. Stoof, Phys. Rev. A 99, 013625 (2019).

[27] F. Iemini, A. Russomanno, J. Keeling, M. Schirò, M. Dalmonte, and R. Fazio, Phys. Rev. Lett. 121, 035301 (2018).

[28] L. Papariello, O. Zilberberg, A. Eichler, and R. Chitra, Phys. Rev. E 94, 022201 (2016).

[29] A. Leuch, L. Papariello, O. Zilberberg, C. L. Degen, R. Chitra, and A. Eichler, Phys. Rev. Lett. 117, 214101 (2016).

[30] A. Eichler, T. L. Heugel, A. Leuch, C. L. Degen, R. Chitra, and O. Zilberberg, Appl. Phys. Lett. 112, 233105 (2018).

[31] E. Goto, Proc. IRE 47, 1304 (1959).

[32] J. Woo and R. Landauer, IEEE J. Quantum Electron. 7, 435 (1971).

[33] I. Mahboob and H. Yamaguchi, Nat. Nanotechnol. 3, 275 (2008).

[34] I. Mahboob, E. Flurin, K. Nishiguchi, A. Fujiwara, and H. Yamaguchi, Nat. Commun. 2, 198 (2011).

[35] I. Mahboob, M. Mounaix, K. Nishiguchi, A. Fujiwara, and H. Yamaguchi, Sci. Rep. 4, 4448 (2014). 
[36] H. Goto, Sci. Rep. 6, 21686 (2016).

[37] S. E. Nigg, N. Lörch, and R. P. Tiwari, Sci. Adv. 3, e1602273 (2017).

[38] S. Puri, C. K. Andersen, A. L. Grimsmo, and A. Blais, Nat. Commun. 8, 15785 (2017).

[39] H. Goto, Z. Lin, and Y. Nakamura, Sci. Rep. 8, 7154 (2018).

[40] Z. Wang, A. Marandi, K. Wen, R. L. Byer, and Y. Yamamoto, Phys. Rev. A 88, 063853 (2013).

[41] P. L. McMahon, A. Marandi, Y. Haribara, R. Hamerly, C. Langrock, S. Tamate, T. Inagaki, H. Takesue, S. Utsunomiya, K. Aihara, R. L. Byer, M. M. Fejer, H. Mabuchi, and Y. Yamamoto, Science 354, 614 (2016).

[42] T. Inagaki, K. Inaba, R. Hamerly, K. Inoue, Y. Yamamoto, and H. Takesue, Nat. Photonics 10, 415 (2016).

[43] T. Inagaki, Y. Haribara, K. Igarashi, T. Sonobe, S. Tamate, T. Honjo, A. Marandi, P. L. McMahon, T. Umeki, K. Enbutsu, O. Tadanaga, H. Takenouchi, K. Aihara, K.-i. Kawarabayashi, K. Inoue, S. Utsunomiya, and H. Takesue, Science 354, 603 (2016).

[44] D. Ryvkine and M. I. Dykman, Phys. Rev. E 74, 061118 (2006).

[45] H. B. Chan, M. I. Dykman, and C. Stambaugh, Phys. Rev. Lett. 100, 130602 (2008).

[46] Z. R. Lin, Y. Nakamura, and M. I. Dykman, Phys. Rev. E 92 , 022105 (2015).

[47] J. Guckenheimer and P. Holmes, Nonlinear Oscillations, Dynamical Systems, and Bifurcations of Vector Fields (Springer, New York, 1983).
[48] C. Zerbe, P. Jung, and P. Hänggi, Phys. Rev. E 49, 3626 (1994).

[49] A. Itô, Prog. Theor. Phys. 61, 815 (1979).

[50] K. Tomita, Phys. Rep. 86, 113 (1982).

[51] S. Bishop, A. Sofroniou, and P. Shi, Chaos, Solitons Fractals 25, 257 (2005).

[52] See Supplemental Material at http://link.aps.org/ supplemental/10.1103/PhysRevLett.123.124301 for derivation of $\lambda_{\text {th }}^{s / a}$ and description of the calibration measurements.

[53] P. Danzl and J. Moehlis, Nonlinear Dyn. 59, 661 (2010).

[54] M. I. Dykman, C. M. Maloney, V. N. Smelyanskiy, and M. Silverstein, Phys. Rev. E 57, 5202 (1998).

[55] H. B. Chan and C. Stambaugh, Phys. Rev. Lett. 99, 060601 (2007).

[56] F. Minganti, N. Bartolo, J. Lolli, W. Casteels, and C. Ciuti, Sci. Rep. 6, 26987 (2016).

[57] N. Bartolo, F. Minganti, W. Casteels, and C. Ciuti, Phys. Rev. A 94, 033841 (2016).

[58] M. Elliott and E. Ginossar, Phys. Rev. A 94, 043840 (2016).

[59] T. L. Heugel, M. Biondi, O. Zilberberg, and R. Chitra, arXiv:1901.03232.

[60] Y. Zhang, J. Gosner, S. M. Girvin, J. Ankerhold, and M. I. Dykman, Phys. Rev. A 96, 052124 (2017).

[61] I.-M. Svensson, A. Bengtsson, P. Krantz, J. Bylander, V. Shumeiko, and P. Delsing, Phys. Rev. B 96, 174503 (2017).

[62] I.-M. Svensson, A. Bengtsson, J. Bylander, V. Shumeiko, and P. Delsing, Appl. Phys. Lett. 113, 022602 (2018). 